Revista Colombiana de Obstetricia y Ginecología Vol. 57 No. 1 • 2006 •(36-44)

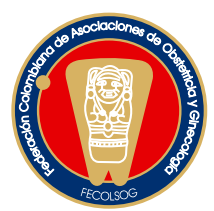

Artículo de Revisión

\title{
CARACTERIZACIÓN CLÍNICA Y BIOQUÍMICA DE LA MUJER CON SÍNDROME DEL OVARIO POLIQUÍSTICO
}

\section{Clinical and biochemical characterization \\ of women having polycystic ovary syndrome}

\author{
Carlos Alfonso Builes, M.D.*, Ivonne Diaz, M.D.**, Jimmy Castañeda, M.D.**, \\ Luis Ernesto Pérez, M.D.***
}

Recibido: enero 24/2006 - Revisado: febrero 23/2006 - Aceptado: marzo 17/2006

\section{RESUMEN}

El Síndrome de Ovario Poliquístico (SOP) tiene una prevalencia entre 4 y $8 \%$ en la población general joven (18 a 45 años de edad). Su diagnóstico se hace con la presencia de dos de los siguientes tres criterios: oligo-anovulación, anovulación, hiperandrogenismo clínico o asintomático y morfología poliquística del ovario al ultrasonido. En el estudio del SOP se deben descartar otras causas de hiperandrogenismo como hiperplasia adrenal congénita, síndrome de Cushing y tumores productores de andrógenos. Las pacientes con SOP tienen alto riesgo de desarrollar intolerancia a los carbohidratos, diabetes y síndrome metabólico, pero no existe evidencia clínica contundente que respalde en ellas una mayor incidencia de eventos isquémicos del miocardio, ni de cáncer endometrial.

* Especialista en medicina interna Universidad de AntioquiaEndocrinología Universidad Militar Nueva Granada. Correo electrónico: cabuba493@gmail.com.

** Especialista en ginecología y obstetricia. Servicio de Ginecología. Unidad de Infertilidad y Endocrinología Reproductiva, Hospital Militar Central y Unidad de Fertilidad Procreación Médicamente Asistida de la Clínica Marly.

*** Especialista en ginecología y obstetricia. Docente Universidad Militar Nueva Granada.
Palabras clave: síndrome del ovario poliquístico, hirsutismo, hiperandrogenismo

\section{SUMMARY}

Polycystic Ovary Syndrome (PCOS) has 4\%-8\% prevalence in young people. Diagnosis is based on two out of the following three criteria: oligoanovulation, clinical or biochemical hyperandrogenism and polycystic morphology using ultrasound. It is necessary to rule out other hyperandrogenic entities like congenital adrenal hyperplasia, Cushing's disease and androgen-producing tumors.

PCOS patients have a high risk of carbohydrate intolerance, diabetes and metabolic syndrome, but there is no strong medical evidence to support greater ischemic myocardial events or endometrial cancer frequency in such patients.

Key words: polycystic ovary syndrome, hirsutism, hyperandrogenism

\section{INTRODUCCIÓN}

En 1935 Stein y Leventhal publicaron su descripción de siete mujeres con amenorrea, hirsutismo, obesidad 
y la apariencia característica de ovarios poliquísticos. Esta fue una de las primeras descripciones de una entidad frecuente y heterogénea en su forma de presentación. ${ }^{1}$ Aunque se han propuesto varias causas del SOP: incremento en la pulsatilidad hipofisiaria de hormona luteinizante(LH) y mayor producción ovárica de andrógenos, aumento de la insulina circulante secundario a resistencia a la insulina y fosforilación del residuo serina del receptor de insulina y mayor respuesta adrenal ante el estímulo, se considera que su origen es multifactorial y complejo. (Figura 1)

La prevalencia del SOP en mujeres premenopáusicas es de 4\% a 8\% entre los 18 y 45 años de edad y $35 \%$ a $40 \%$ en mujeres infértiles, constituyéndose en una de las más importantes y comunes anormalidades endocrinológicas de la mujer. ${ }^{2-6}$

\section{Criterios diagnósticos}

El SOP puede tener manifestaciones clínicas generales, endocrinológicas y metabólicas, constituyendo un amplio espectro a lo largo de la vida. (Figura 2). En general el diagnóstico de SOP se basa principalmente en la historia clínica, pero en la actualidad basta con la presencia de dos de los siguientes tres criterios propuestos en el 2003 en la reunión de Rotterdam: ${ }^{7}$

1. Oligoovulación o anovulación.

2. Niveles elevados de andrógenos circulantes o manifestaciones clínicas de exceso de andrógenos ováricos.

3. Morfología de ovarios poliquísticos definida por ecografía.

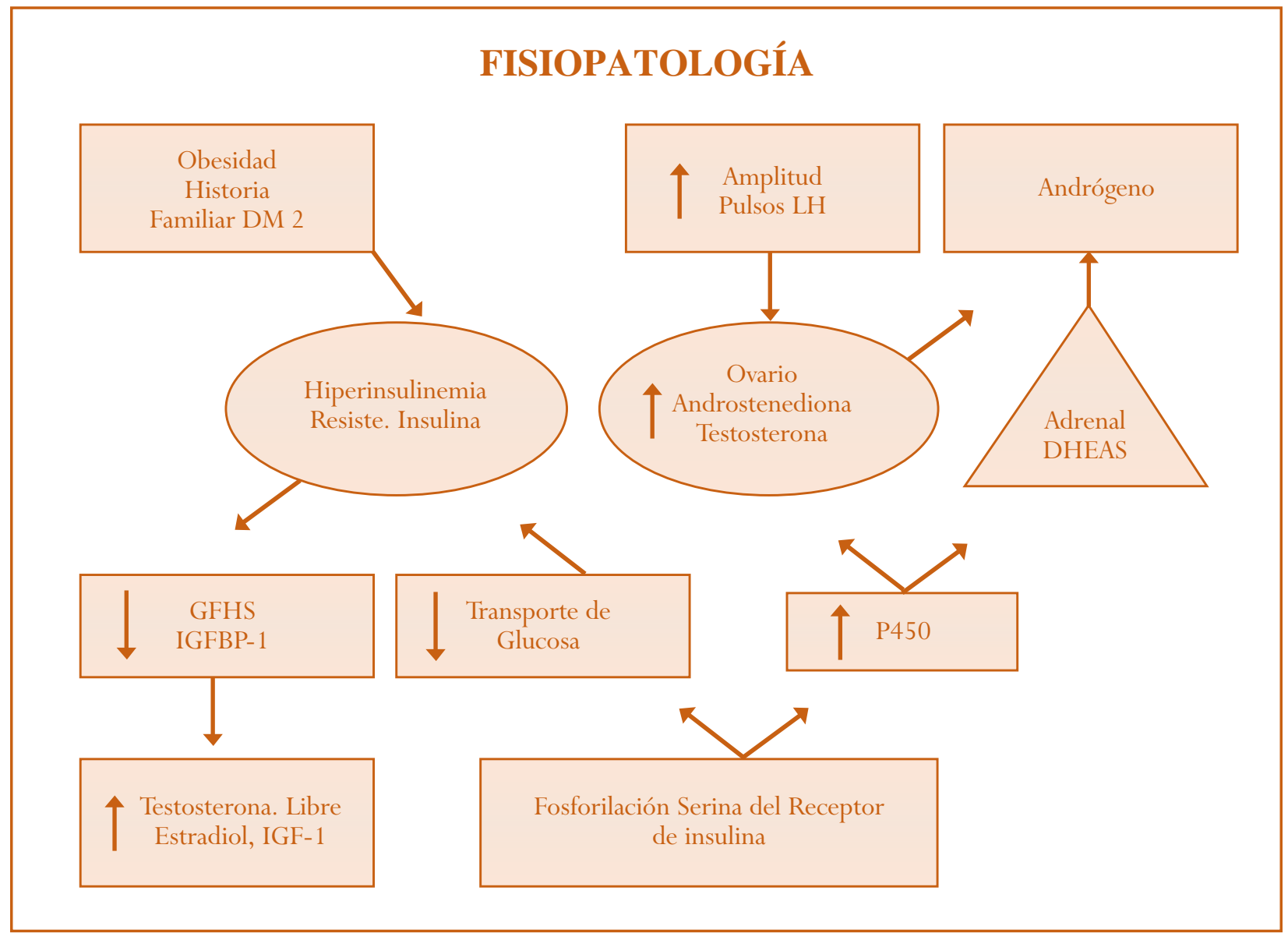

Figura 1. DM2: Diabetes Mellitus, LH: hormona luteinizante, GFHS:Globulina Fijadora de hormonas sexuales, Proteina ligadora del factor de crecimiento insulinoide 1, IGF-1: Factor de crecimiento insulinoide 1, P450: Citocromo P450, DHEA: Dehidroepiandrosterona sulfato. 


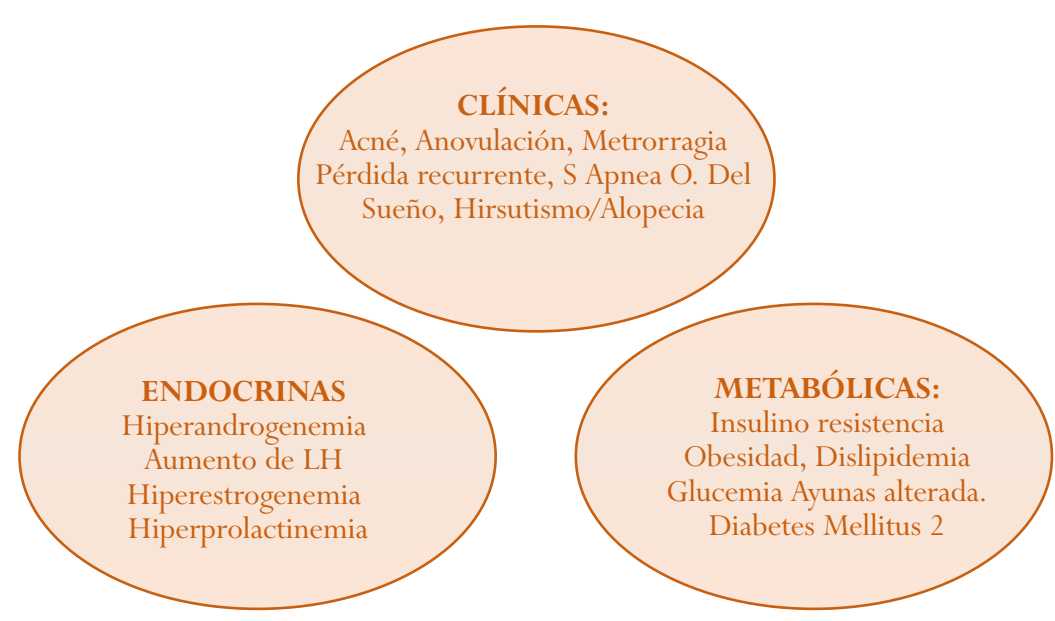

Figura 2.

\section{Oligoovulación o anovulación}

Los trastornos del ciclo se manifiestan frecuentemente como:

a. Oligomenorrea: Ciclos mayores de 35 días o la presencia de menos de 9 ciclos en un año. En las mujeres con ciclos regulares cerca de 3,7\% tienen anovulación. ${ }^{8}$

b. Niveles de progesterona en la fase lútea media (día 21) menores de $15 \mathrm{ng} / \mathrm{ml}$.

c. Presencia de hemorragia uterina anormal, no precedida de síntomas premenstruales.

d. Infertilidad.

\section{Niveles elevados de andrógenos circulantes o manifestaciones clínicas de exceso de andrógenos}

Las manifestaciones cutáneas del hiperandrogenismo en el SOP incluyen hirsutismo, piel grasosa/acné y alopecia de patrón masculino. La escala de medición de hirsutismo más utilizada es la de FerrimanGallwey que califica la presencia de vello terminal en 9 áreas corporales: supralabial, mentón, tórax, abdomen superior e inferior, dorso superior e inferior, muslos y brazos; con puntaje de 0 a 4 , considerándose positiva la presencia de un puntaje mayor de 8. Esta escala presenta una alta variabilidad interobservador. ${ }^{9}$

No se cuenta con valores locales de referencia, ajustados por edad, peso y raza para los andrógenos en nuestra población, sin embargo, valores $>200$ $\mathrm{ng} / \mathrm{dl}$ de testosterona total y $>7000 \mathrm{ng} / \mathrm{ml}$ de dehidroepiandrosterona (DHEAS) sugieren la presencia de una neoplasia productora de andrógenos.

\section{Morfología de ovario poliquístico definido por ecografía}

No se requiere la presencia del ovario poliquístico para hacer el diagnóstico de SOP y su presencia aislada tampoco lo establece. ${ }^{10}$ Se ha descrito la morfología poliquística en 23\% de las mujeres premenopáusicas. ${ }^{11}$ El estudio de Adams y cols. en mujeres con ciclos ovulatorios normales y morfología de ovario poliquístico, halló un incremento en los niveles de andrógenos y menores niveles de globulina fijadora de hormonas sexuales respecto a mujeres con ciclos ovulatorios normales sin morfología poliquística, dejando abierta la discusión acerca de la existencia de una entidad que representa la forma más leve de hiperandrogenismo ovárico ${ }^{12}$ 
En el estudio ecográfico del ovario poliquístico, se requiere que la imagen sea obtenida en la fase folicular temprana (día 3-5 del ciclo). Dicha imagen debe mostrar crecimiento ovárico con 12 o más folículos antrales que varían de 2 a $10 \mathrm{~mm}$ en diámetro, organizados en distribución periférica y central e incremento del estroma central mayor del 25\% del área ovárica.$^{13}$ En caso de haber un folículo dominante (>10 mm) o un cuerpo lúteo, el ultrasonido debería repetirse durante el siguiente ciclo. El diagnóstico diferencial se hace con el ovario multifolicular, en el cual el tamaño es normal o discretamente incrementado, contiene 6 o más folículos, sin distribución periférica ni aumento del estroma central.

El SOP es un síndrome de presentación heterogénea como lo demostró Azziz y colaboradores, quienes evaluaron 400 mujeres, no seleccionadas, encontrando $73 \%$ de pacientes eumenorreicas sin hirsutismo, 20\% con disfunción menstrual únicamente (con SOP confirmado en 8\%), 4\% con hirsutismo solamente (con SOP confirmado en 67\%) y 2,8\% con disfunción menstrual e hirsutismo (con SOP confirmado en 86\%). De las pacientes con SOP confirmado, $75 \%$ tenían hirsutismo. ${ }^{4}$

\section{Diagnóstico diferencial}

Antes de confirmar el diagnóstico de SOP se deben excluir otras entidades que pueden semejar algunas manifestaciones clínicas: (Tabla 1)

\section{Hiperplasia suprarrenal congénita (HSC)}

Esta es una hiperplasia de variedad no clásica, con deficiencia parcial de la 21 -hidroxilasa, un desorden genético de herencia autosómica recesiva y cuyo marcador es el incremento de la 17 -hidroxiprogesterona (17 OHP). Puede asociarse con un incremento en la producción de androstenediona y testosterona con signos de hiperandrogenismo, presentando hirsutismo severo, clitoromegalia, menstruaciones regulares, herencia familiar y talla baja.

Se debe hacer medición de la 17 OHP en la fase folicular temprana (día 3 al 5 del ciclo). Son valores confirmatorios de HAC, una 17 OHP basal mayor de $4 \mathrm{ng} / \mathrm{ml}$ o $>10 \mathrm{ng} / \mathrm{ml} 60$ minutos posestímulo con $250 \mu \mathrm{g}$ de corticotrofina (ACTH) intravenosa. Los valores basales menores de $2 \mathrm{ng} / \mathrm{ml}$ prácticamente descartan este desorden, mientras que valores

\begin{tabular}{|c|c|c|c|c|c|c|c|c|c|}
\hline Enfermedad & Hirsutismo & Anovulación & 17 OHP & E2 & Test & DHEAS & LH & FSH & MOP \\
\hline HAC & + & + & MA & A & A & A & A & $\mathrm{No} \mathrm{B}$ & $82 \%$ \\
\hline Tumor Adrenal & + & + & A & $\mathrm{NoA}$ & MA & MA & N-A & N-B & $25 \%$ \\
\hline Tumor Ovárico & ++ & + & $\mathrm{N}$ & MA & MA & A & N-B & N-B & $80 \%$ \\
\hline Sind. Cushing & + & + & $\mathrm{N}$ & N-B & N-B & N-A-B & N-A-B & N-B & $46 \%$ \\
\hline Acromegalia & + & + & $\mathrm{N}$ & N-B & $\mathrm{N}$ & $\mathrm{N}$ & N-A-B & N-B & --- \\
\hline Hiperprolactinemia & + & + & $\mathrm{N}$ & N-B & $\mathrm{N}-\mathrm{A}$ & A & N-B & N-B & $50-67 \%$ \\
\hline DM 2 & + & + & $\mathrm{N}$ & $\mathrm{N}$ & N-A & $\mathrm{N}$ & N-A & $\mathrm{N}$ & $68-82 \%$ \\
\hline \multicolumn{10}{|c|}{$\begin{array}{l}17 \text { (OH) P: } 17 \text { Hidroxi progesterona. E2: Estradiol; Test: Testosterona; DHEAS: Dehidro epi androsterona sulfato; LH: hor- } \\
\text { mona Luteinizante; FSH: Hormona Folículo estimulante, MOP: Morfología de ovario poliquístico en la ultrasonografía. HAC: } \\
\text { Hiperplasia Adrenal Congénita; DM2: Diabetes Mellitus 2. MA: Muy aumentado; A: Aumentado; N: Normal, B: bajo. }\end{array}$} \\
\hline
\end{tabular}


entre 2 y 4 ameritan confirmación con la prueba de estímulo con ACTH. ${ }^{14,15}$

La deficiencia de 11 -hidroxilasa es la deficiencia enzimática suprarrenal productora de hiperplasia que ocupa el segundo lugar en frecuencia. Puede acompañarse de hirsutismo moderado e hipertensión arterial y elevación de 17 OHP y 11 deoxicortisol.

\section{Síndrome de Cushing}

La redistribución grasa, especialmente en los cojinetes supraclaviculares, la piel delgada con presencia de equimosis o estrías purpúricas mayores de $1 \mathrm{~cm}$ de ancho, sugieren una alta probabilidad de esta patología. Otros signos clínicos son la facies de luna llena, obesidad, hirsutismo, acné, irregularidad menstrual, joroba de búfalo, hipertensión arterial y piernas delgadas para el volumen aumentado del tronco. Los ovarios en la mayoría de pacientes no tienen apariencia poliquística. Se determina la presencia de hipercortisolismo con un incremento del cortisol en orina de 24 horas ( $>2$ veces el rango de referencia) o cortisol sérico a las 8 a.m. que no haya sido suprimido, luego de la administración de $1 \mathrm{mg}$ de dexametasona la noche anterior a las 11 p.m.: $>1,8 \mu \mathrm{g} / \mathrm{dl}$ ó $>50 \mathrm{nmol} / \mathrm{L} .{ }^{16}$

\section{Neoplasias ováricas o suprarrenales virillizantes}

Estas neoplasias cursan con una progresiva y rápida regresión de caracteres femeninos (desfeminización) y posterior presentación de hirsutismo severo, clitoromegalia, alopecia de patrón masculino, incremento de la libido, voz gruesa y acné (masculinización). La disfunción menstrual puede variar desde sangrado irregular hasta amenorrea y en algunos pacientes se puede palpar una masa pélvica o abdominal que sugieren tumor. En estos pacientes es importante tener en cuenta los marcadores tumorales, la dehidroepiandrosterona sulfato (DHEA-S) el cual es por excelencia el marcador de andrógenos de origen suprarrenal y la testosterona, de origen gonadal.

\section{Hipertecosis ovárica}

Este tipo de pacientes cursan con valores elevados de andrógenos e insulina y signos de virilización. A menudo son obesas y manifiestan acantosis pigmentaria.

Es una condición proliferativa inusual en la cual el ovario contiene nidos de células de la teca, luteinizadas dispersas a través del estroma. El ovario se encuentra aumentado de tamaño y con textura firme como consecuencia del crecimiento denso de fibroblastos. La ausencia de la formación de folículos lo diferencia del ovario hallado en SOP.

\section{Acromegallia}

Aunque esta patología puede producir signos moderados de hiperandrogenismo y alteración de los ciclos menstruales, predominan las manifestaciones de crecimiento en manos y/o pies, separación dental, rasgos faciales gruesos con región supraciliar prominente y base de la nariz ancha. Se diagnostica midiendo la hormona de crecimiento (HC) a 1 y 2 horas luego de la administración de 75 gramos de glucosa, si no se logra suprimir a $<$ $1 \mu \mathrm{g} / \mathrm{L}$ la $\mathrm{HC}$ y/o con niveles elevados de somatomedina $\mathrm{C}(\mathrm{IGF}-1) .{ }^{17}$

\section{Hiperprolactinemia}

Esta condición causa el 30\% de las amenorreas secundarias en mujeres de edad reproductiva, produciendo hipogonadismo hipogonadotrópico. En el SOP puede presentarse hiperprolactinemia no tumoral asociada al estímulo estrogénico persistente, hasta en $35 \%$ de los casos. ${ }^{18}$

Existen receptores ováricos para prolactina que favorecen la producción androgénica. La presencia de oligoamenorrea o amenorrea y galactorrea sugieren hiperprolactinemia, la cual es confirmada con medición sérica de prolactina, tomada en ayunas, guardando reposo previo al análisis de sangre y evitando la noche anterior cualquier estímulo sobre la pared torácica. ${ }^{19}$ La presencia de valores mayores de $100 \mathrm{ng} / \mathrm{ml}$ son altamente sugestivos de adenoma hipofisiario. ${ }^{20}$ 


\section{Otros}

a) Hipotiroidismo primario: Puede causar irregularidades menstruales (amenorrea) e infertilidad anovulatoria. ${ }^{21}$ Se debe sospechar en presencia de bocio, edema palpebral inferior y fase de relajación lenta del reflejo aquiliano, TSH elevada y T3 y T4 bajos. Los niveles bajos de T3 estimulan la producción de tiroliberina (TRH) (hormona liberadora de tirotropina) la cual estimula tanto la tirotropina (TSH), como prolactina, explicando así el hallazgo de hiperprolactinemia en algunos casos de hipotiroidismo primario. ${ }^{22,23}$

b) Falla ovárica prematura: Cursa con amenorrea por mas de tres ciclos, con disminución de la reserva ovárica y con hipogonadismo hipergonadotrópico (niveles de FSH > de $40 \mathrm{UI} / \mathrm{ml}$ y niveles bajos de estradiol $<50 \mathrm{pg} / \mathrm{ml}$ ). La falla ovárica prematura no se acompaña de signos de hiperandrogenismo y puede manifestarse en el contexto de otros desordenes autoinmunnitarios, especialmente insuficiencia suprarrenal, hipotiroidismo y anemia perniciosa. ${ }^{24,25}$

c) Obesidad: Puede acompañarse de signos de resistencia periférica a la insulina (acantosis pigmentaria), dislipidemia e hipertensión arterial. No se acompaña con frecuencia, de oligomenorrea o amenorrea. El aumento del perímetro abdominal (mayor de $90 \mathrm{~cm}$ para hombre y mayor de $80 \mathrm{~cm}$ para las mujeres ${ }^{26}$ se constituye en el criterio principal del diagnóstico de síndrome metabólico junto con al menos dos de los siguientes: glucemia basal $>100 \mathrm{mg} / \mathrm{dl}$, colesterol HDL $<40 \mathrm{mg} / \mathrm{dl}$ en hombres o $<$ de $50 \mathrm{mg} / \mathrm{dl}$ en mujeres, triglicéridos $>150 \mathrm{mg} / \mathrm{dl}$ y cifras de presión arterial $>$ 130/85 $\mathrm{mmHg}$.

La acantosis pigmentaria se considera como un marcador de hiperinsulinemia y surge de la hiperqueratosis epidérmica y proliferación dérmica de fibroblastos. ${ }^{27}$

d) Medicamentos: el uso de andrógenos, ciclosporina y ácido valproico pueden producir signos de hiperandrogenismo. ${ }^{28}$

\section{Evaluación del laboratorio}

La evaluación endocrinológica mínima sugerida es la medición de testosterona total, DHEAS y 17 OHP. La principal razón de medir las dos primeras es la exclusión de un tumor productor de andrógenos. Como parte de la evaluación de oligomenorrea y ante la sospecha clínica, debe medirse TSH y prolactina séricas.

La medición de niveles de gonadotropinas (relación $\mathrm{LH} / \mathrm{FSH}$ ) no se recomienda, ya que esta no da información adicional al diagnóstico y cerca de una tercera parte de pacientes pueden tener LH en rango normal. La testosterona libre tiene su principal utilidad en el monitoreo de la eficacia del tratamiento para la reducción de la producción de andrógenos.

La evaluación bioquímica de resistencia a la insulina no parece ser esencial en el diagnóstico, ni se dispone de validación local. La relación glucemia/ insulinemia basales $<4,5$, identificó resistencia a insulina con sensibilidad de 95\% y especificidad de $84 \%$ con valor predictivo positivo de $87 \%$ y predictivo negativo de $94 \% .^{29}$

Se recomienda hacer medición del perfil de lípidos en pacientes obesos con SOP, pues la hiperandrogenemia, hiperinsulinemia y obesidad ejercen en forma independiente efectos adversos sobre el perfil de lípidos.

La ecografía de útero y anexos además de evaluar la morfología ovárica, permite la determinación de hiperplasia endometrial. Se recomienda la vía transvaginal, especialmente en pacientes obesas y en días 3 al 5 del ciclo. En mujeres con oligomenorrea debería hacerse al azar o entre días 3 al 5 luego de sangrado inducido con progestágenos.

La tomografía axial computarizada permite una visualización adecuada de las glándulas suprarrenales ante la sospecha de lesión neoplásica.

\section{SOP y sus asociaciones}

Los síntomas del síndrome del ovario poliquístico usualmente comienzan alrededor de la menarquia, pero su inicio después de la pubertad puede propiciarse por modificaciones medioambientales como el aumento de peso. El antecedente de pubarquia prematura, la cual puede ser resultado de una secreción temprana de esteroides por las glándulas suprarrenales puede ser una forma heraldo del síndrome. 
Se ha encontrado que niños con antecedente de restricción del crecimiento intrauterino son más propensos a desarrollar hiperinsulinismo, pubarquia prematura y signos de SOP en la vida reproductiva. ${ }^{30,31}$

\section{Obesidad}

La incidencia de obesidad en mujeres con SOP varía entre países y grupos étnicos. La frecuencia de obesidad en pacientes con SOP es más alta respecto a la población general estadounidense (42\% contra 32\%) y hay mayor prevalencia de obesidad en mujeres estadounidenses que en mujeres españolas con SOP (42\% contra 30\%).

\section{Intolerancia a la glucosa}

Las mujeres con SOP tienen un mayor riesgo de desarrollar intolerancia a la glucosa, diabetes mellitus tipo $2^{32,33}$, dislipidemia (HDL disminuido $<50 \mathrm{mg} / \mathrm{dl}$ e hipertrigliceridemia $>150 \mathrm{mg} / \mathrm{dl}$ ), ateroesclerosis y síndrome metabólico. Lo anterior es especialmente importante en pacientes obesas aunque el fenómeno de resistencia a la insulina ha sido descrito también en pacientes no obesas con SOP. ${ }^{34}$

Apridonidze $^{35}$ en 106 pacientes con SOP, encontró 43\% de mujeres con síndrome metabólico (según los criterios de la ATP III). Siendo 7,5 veces más frecuente respecto a la población general de 20 a 29 años $(44,8 \%$ contra $5,9 \%)$ y 3,6 veces más frecuente entre los 30 y 39 años (53\% contra 14,6\%). Además, glucemia en ayunas alterada $(>$ de $100 \mathrm{y}<126 \mathrm{mg} / \mathrm{dl}$ ) en $10 \%$ de las pacientes, intolerancia a los carbohidratos en $11 \%$ y diabetes mellitus tipo 2 en el $8 \% .^{32}$

Además del perfil lipídico de riesgo, se ha descrito aumento en los niveles del PAI-1 (inhibidor del activador del plasminógeno 1), sugiriendo un proceso inflamatorio crónico subyacente.

\section{Enfermedad Cardiovascular}

Las manifestaciones metabólicas del SOP han exaltado inquietudes acerca del riesgo de enfermedad cardiovascular.
A pesar de la fuerte asociación de SOP y factores de riesgo cardiovascular, el estudio de Pierpoint, con seguimiento de 786 mujeres con SOP no mostró exceso de mortalidad de enfermedades circulatorias. Wild no reportó exceso de morbilidad o mortalidad por arteriopatía coronaria en mujeres con SOP y edad media, la mayoría entre 25 y 40 años. Sin embargo, la morbilidad y mortalidad por diabetes y riesgo de enfermedad cerebrovascular no mortal, estuvieron incrementadas en mujeres con SOP. ${ }^{36}$ Esta asociación permanece aún bajo investigación.

\section{Hiperplasia Endometrial y Cáncer Endometrial}

La acción estrogénica sin oposición y la ausencia de menstruación normal inducida por progestágenos podría producir hiperplasia endometrial y hemorragia. Aunque existe el riesgo teórico y estudios previos sugerían un incremento de 4 veces del riesgo de carcinoma endometrial, estudios mas recientes han puesto en duda este dogma, pues no se dispone de una evidencia médica contundente de estudios comparativos que soporten esta afirmación. ${ }^{37}$

\section{CONCLUSIONES}

EL SOP es una entidad frecuente en mujeres de edad reproductiva, para la cual se deben tener en mente otras entidades productoras de hiperandrogenismo en el diagnóstico diferencial. El SOP hace parte de un espectro más amplio de alteraciones metabólicas que deben ser evaluadas en forma temprana como obesidad, dislipidemia, glucemia alterada y síndrome metabólico.

\section{REFERENCIAS}

1. Stein IF, Leventhal ML. Amenorrhea associated with bilateral polycystic ovaries. Am J Obstet Gynecol 1935;29:181-91.

2. Knochenhauer ES, Key TJ, Hahsar-Miller M, Waggoner W, Boots LR, Azziz R. Prevalence of the polycystic ovary syndrome in unselected black and white women of southeastern United States: a prospective study. J 
Clin Endocrinol Metab 1998;83:3078-82.

3. Asuncion M, Calvo RM, San Millan JL, Sancho J, Avila S, Escobar-Morreale HF. A prospective study of the prevalence of polycystic ovary syndrome in unselected Caucasian women from Spain. J. Clin Endocrinol Metab 2000;85:2434-8.

4. Azziz R, Woods KS, Reyna R, Key TJ, Knochenhauer ES, Yildiz BO. The prevalence and features of the polycystic ovary syndrome in an unselected population. J Endocrinol Metab 2004;89:2745-9.

5. Michelmore KF, Balen AH, Dunger DB, Vessey MP. Polycystic ovaries and associated clinical and biochemical features in young women. Clin Endocrinol. (oxf) 1999;51:779-86.

6. Saleh AM, Khalil HS. Review of nonsurgical and surgical treatment and the role of insulin-sensitizing agents in the management of infertile women with polycystic ovary syndrome. Acta Obstet Gynecol Scan 2004;83:614-21.

7. Rotterdam ESHRE/ASRM- Sponsored PCOS Consensus Workshop Group. Revised 2003 consensus on diagnostic criteria and long-term health risks related to polycystic ovary syndrome. Fertil Steril 2004;81:19-25.

8. Malcolm CE, Cumming DC. Does anovulation exist in eumenorrheic women?. Obstet Gynecol 2003;102:317-8.

9. Wild R, Vesely S, Beebe L, Whitsett T, Owen W. Ferriman Gallwey self-scoring I: performance assessment in women with polycystic ovary syndrome. J. Clin Endocrinol Metab 2005;90:4112-4.

10. Ehrman D. Polycystic ovary syndrome. N Engl J Med 2005;352:1223-36

11. Polson DW, Adams J, Wadsworth J, Franks S. Polycystic ovaries-- a common finding in normal women. Lancet 1988;1:870-2.

12. Adams J, Taylor A, Crowley WF Jr, Hall JE. Polycystic ovarian morphology with regular ovulatory cycles insights into the pathophysiology of polycystic ovarian syndrome. J Clin Endocrinol Metab 2004;89:4343-50.

13. Jonard S, Robert Y, Cardet-Rudelli C, Pigny P, Decanter C, Dewailly D. Ultrasound examination of polycystic ovaries: is it worth counting the follicles? Hum Reprod 2003;18:598-603.

14. Dewailly D. Nonclassic 21-hidroxilase deficiency. Semin Reprod Med 2002;20:243-8.

15. Moran C, Azziz R. 21-hydroxylase- deficient nonclassic adrenal hyperplasia: the great pretender. Semin Reprod
Med 2003;21:295-300.

16. Raff H, Findling JW. A physiologic approach to diagnosis of the Cushing syndrome. Ann Intern Med 2003;138:980-91.

17. Growth Hormone Society: Pituitary Society. Biochemical assessment and long-term monitoring in patients with acromegaly: statement from a joint consensus conference of the Growth Hormone Research Society and the Pituitary Society. J Clin Endocrinol Metab 2004;89:3099-102.

18. Pérez LE. Síndrome de Ovario poliquístico. Patologías asociadas a hirsutismo. En: Pérez LE. Infertilidad y Endocrinología Reproductiva. 2a. ed. Bogotá, Colombia: Imprenta Hospital Militar; 2000. p. 152-61.

19. Schlechte J. Clinical practice. Prolactinoma. N Engl J Med 2003;349:2035-41.

20. Bayrak A, Saadat P, Mor E, Chong R, Paulson RJ, Sokol RZ. Pituitary imaging is indicated for the evaluation of hyperprolactinemia. Fertil Steril 2005;84:181-5.

21. Kalro BN. Impaired fertility caused by endocrine dysfunction in women. Endocrinol Metab Clin North Am 2003;32:573-92.

22. García J, Polanía D, Builes CA. Hiperplasia hipofisiaria secundaria a hipotiroidismo primario. Acta Med Colomb 2005;30:65-7.

23. Ozbey N, Sariyildiz E, Yilmaz L, Orthan Y, Sencer E, Molvalilar S. Primary hypothyroidism with hyperprolactinaemia and pituitary enlargement mimicking a pituitary macroadenoma. Int J Clin Pract 1997;51:409-11.

24. Nelson LM, Covington SN, Rebar RW. An update: spontaneous premature ovarian failure is not an early menopause. Fertil Steril 2005;83:1327-32.

25. Padrón R, Sell J, Montejo L. Búsqueda de trastornos endocrinos en pacientes con falla ovárica prematura. Rev Cubana Endocrinol 2001;12:144-9.

26. The IDF consensus world wide definition of metabolic syndrome. 2005. http://www.IDF.org

27. Chang RJ. A practical approach to the diagnosis of polycystic ovary syndrome. Am J Obstet Gynecol 2004;191:713-7.

28. Isojarvi JL, Laatikainen TJ, Pakarinen AJ, Juntunen KT, Myllyla VV. Polycystic ovaries and hyperandrogenism in women taking valproato for epilepsy. N Engl J Med 1993;329:1383-8.

29. Legro RS, Finegood D, Dunaif A. A fasting glucose to insulin ratio is a useful measure of insulin sensitivity 
in women with polycystic ovary syndrome. J Clin Endocrinol Metab 1998;83:2694-8.

30. Norman RJ, Wu R, Stankiewicz M. Polycystic ovary syndrome. Med J Aust 2004;180:132-7.

31. Ibanez L, Polau N, de Zegher F. Ovarian hyperresponsiveness to follicle stimulating hormone in adolescent girl born small for gestational age. J Clin Endocrinol Metab 2000;85:2624-6.

32. Colilla S, Cox NJ, Ehrmann DA. Heritability of insulin secretion and insulin action in women with polycystic ovary syndrome and their first degree relatives. J Clin Endocrinol Metab 2001;86:2027-31.

33. Legro RS, Kunselman AR, Dodson WC, Dunaif A. Prevalence and predictors of risk for type 2 diabetes mellitus and impaired glucose tolerance in polycystic ovary syndrome: a prospective, controlled study in 254 affected women. J Clin Endocrinol Metab 1999;84:165-9.
34. Dunaif A, Segal KR, Futterweit W, Dobrjaosky A. Profound peripheral insulin resistance, independent of obesity, in polycystic ovary syndrome. Diabetes 1989;38:1165-74.

35. Apridonidze T, Essah P, Inorno M,. Prevalence and characteristics of the metabolic syndrome in women with polycystic ovary syndrome. J Clin Endocrinol Metab 2005;90:1929-35.

36. Wild S, Pierpoint T, McKeigue P, Jacobs H. Cardiovascular disease in women with polycystic ovary syndrome at long term follow up: a retrospective cohort study. Clin Endocrinol (Oxf) 2000;52:595-600.

37. Hardiman P, Pillay OC, Atiomo W. Polycystic ovary syndrome and endometrial carcinoma. Lancet 2003;361:1810-2. 